\title{
Overcoming the Adverse Effects of Everolimus to Achieve Maximum Efficacy in the Treatment of Inoperable Breast Cancer: A Review of 11 Cases at Our Hospital
}

\author{
Yuko Yoshida-Ichikawa ${ }^{a}$ Masahiko Tanabe $^{b} \quad$ Emi Tokuda $^{a}$ \\ Hideo Shimizu $^{a}$ Yoshiya Horimoto $^{a}$ Kayo Miura ${ }^{a}$ Mitsue Saito ${ }^{a}$ \\ aDepartment of Breast Oncology, Juntendo University Hospital, Tokyo, Japan; \\ bDepartment of Breast and Endocrine Surgery, Graduate School of Medicine, \\ The University of Tokyo, Tokyo, Japan
}

\section{Keywords}

Breast cancer · Everolimus · Adverse events - Estrogen receptor · PI3K/Akt/mTOR

\begin{abstract}
Everolimus is an effective treatment for advanced and/or metastatic breast cancer, especially in hormone receptor-positive cases. However, adverse events have prevented considerable numbers of clinicians from using this drug. Herein, we reviewed our clinical experiences and endeavored to identify patients in whom the benefits of everolimus treatment would outweigh these adverse events. If measures were available to prevent or minimize adverse effects prior to treatment, everolimus would be a more widely applicable drug. This retrospective study involved 11 patients in whom nonresectable or recurrent breast cancers were treated with everolimus between April 2014 and January 2016. Two patients achieved a partial response (PR) and 4 showed stable disease (SD) (1 showed long SD, i.e., >24 weeks). The response rate
\end{abstract}


was $18 \%$, and the clinical benefit rate (PR + long SD) was $27 \%$. Regarding adverse events, interstitial pneumonia (grade 3 ) developed in 3 patients (18\%), necessitating treatment discontinuation. When using everolimus, it may be important to select suitable patients for whom this treatment can be continued with sufficient control of adverse events. Herein, we provide information relevant to the clinical use of everolimus based on our daily practice experiences with this agent.

(C) 2018 The Author(s)

Published by S. Karger AG, Basel

\section{Introduction}

Endocrine therapy is the most effective systemic treatment for hormone receptor-positive breast cancer patients. Its efficacy is often limited, however, by the onset of resistance. Several patterns of endocrine resistance are recognizable clinically. The mechanism of action of everolimus involves inhibition of the PI3K/Akt/mTOR pathway. This pathway is activated when tumors develop acquired resistance, which means that a tumor which initially responded to hormone therapy loses this reactivity over the course of several treatments [1]. Constant activation of the PI3K/Akt/mTOR signal pathway, which is involved in differentiation and proliferation of tumor cells, is observed [2] in hormone therapy-resistant breast cancer. Everolimus was approved for the treatment of advanced recurrent breast cancer positive for the estrogen receptor (ER) and negative for the human epidermal growth factor receptor type 2 (HER2) in March 2014 in Japan. Furthermore, everolimus is expected to overcome aromatase inhibitor resistance.

The BOLERO-2 study investigated the efficacy of combining a steroidal aromatase inhibitor, exemestane, with everolimus against ER-positive breast cancer which had recurred and advanced after treatment with a nonsteroidal aromatase inhibitor. Most patients registered in the BOLERO-2 study showed acquired resistance to hormone therapy [3]. Progression-free survival (PFS) was significantly extended in the everolimus combined group but overall survival was not.

Accordingly, based on previous clinical study results, everolimus is considered to be indicated for patients with acquired resistance. However, factors predicting the effectiveness of everolimus have yet to be established. Moreover, adverse events, including interstitial pneumonia and stomatitis, may reportedly discourage patients and/or clinicians from using everolimus continuously [3]. Therefore, considerable numbers of clinicians are unwilling to use this drug, despite its marked therapeutic effects.

We reviewed our clinical experience and examined patients for whom the benefits of everolimus treatment outweighed the adverse events. Herein, we present information on the clinical use of everolimus, based on our daily practice experiences, which may be useful for clinicians considering the administration of this drug to their patients. 


\section{Case Reports in Oncology}

Case Rep Oncol 2018;11:511-520

DOI: $10.1159 / 000490787$

(c)

2018 The Author(s). Published by S. Karger AG, Base www.karger.com/cro

Yoshida-Ichikawa et al.: Overcoming the Adverse Effects of Everolimus to Achieve

Maximum Efficacy in the Treatment of Inoperable Breast Cancer

\section{Subjects and Methods}

This retrospective study involved 11 patients in whom nonresectable or recurrent breast cancers, ER positive and HER2 negative, had been treated between April 2014 and January 2016 with everolimus and exemestane.

Clinical effects and adverse events were retrospectively investigated. ER, progesterone receptor, and HER2 were assessed by immunostaining of surgical or needle biopsy specimens in all patients. As treatment, everolimus at $5-10 \mathrm{mg} /$ day and exemestane at $25 \mathrm{mg} /$ day were concomitantly administered orally. The therapeutic effect was evaluated following the RECIST guidelines (version 1.1). Adverse events were evaluated based on descriptions in the medical records using CTCAE (version 4.0). For blood biochemistry, blood glucose, cholesterol, and triglycerides were measured every 2 weeks until the second cycle after initiation of the regimen and then every cycle thereafter. The presence or absence of pulmonary lesions was confirmed by chest computed tomography (CT) before the introduction of everolimus.

\section{Results}

We retrospectively reviewed the medical records of 11 patients, with nonresectable or recurrent breast cancers, who had been administered everolimus between April 2014 and January 2016. The patient background data are shown in Table 1a. The median age was 58 years (range 48-74). All patients were postmenopausal. Eight patients had recurrent breast cancer, with 3 having stage IV tumors and distant metastasis at the first examination. The stage of the postoperative recurrent cases at the time of surgery was IIA in 2 patients, IIB in 4 patients, IIIA in 1 patient, and IIIC in 1 patient. The median duration of administration was 16 weeks (4-68 weeks). On immunostaining, ER was positive in all patients, while both ER and progesterone receptor were positive in 8 patients. All patients were negative for HER2. The distant metastasis sites at the initiation of everolimus treatment included bone in 7 patients (64\%), and bone was the only metastatic site in 2 patients (18\%) patients. The visceral organs (liver and lungs) harbored metastases in 8 patients (18\%), and visceral organs were the only metastatic site in 3 patients (27\%).

The details of previous treatments in each patient are shown in Figure 1. Nine patients had received chemotherapy before everolimus. All patients had received endocrine therapy before everolimus. In addition, 4 patients had taken exemestane before treatment with everolimus.

None of the patients obtained a complete response. A partial response (PR) was observed in 2 patients, stable disease (SD) in 4 patients (1 patient showed long SD, i.e., $>24$ weeks), progressive disease (PD) in 2 patients, and the disease status could not be determined in 3 patients (Fig. 1). The response rate (RR) was $18 \%$, and the clinical benefit rate (PR + long SD) was $27 \%$. In the 8 patients with postoperative recurrence, the effect was PR in 1 patient, SD in 2 patients (neither showed long SD), PD in 2 patients, and it could not be determined in 3 patients. The RR and the clinically useful rate (PR + long SD) were both $13 \%$. The RR based on the time of recurrence, previous treatment, and recurrent site is shown in Table $1 \mathrm{~b}$. Two of 3 patients had inoperable disease and the outcome was PR or long SD. The duration of the response to previous endocrine therapy was 12 months, i.e., long, in these 2 patients, indicating 
that the drugs had been administered in a favorable state of endocrine therapy sensitivity. Thus, patients who had received several previous treatments ( $>5$ regimens) were included $(7$ patients), and 3 achieved PR or long SD. Patients previously treated with exemestane acquired SD or a better therapeutic effect.

Grades of toxicity are shown in Table 2a. Interstitial pneumonia (grade 3, G3) was observed in 3 patients (18\%). G1 and G2 adverse events were interstitial pneumonia in 1 patient (9\%), glucose metabolic disorder in 2 patients (18\%), lipid metabolic disorder in 2 patients $(18 \%)$, stomatitis in 3 patients (27\%), and thrombocytopenia in 2 patients (18\%). Dose reduction was necessary in 3 patients mainly due to thrombocytopenia (G2), stomatitis (G2), and interstitial pneumonia (G1). Treatment was discontinued in 4 patients (36\%) because of interstitial pneumonia in 3 patients (G3 in all cases) and stomatitis in 1 patient (G2). Moreover, everolimus administration was not restarted in any of these patients. In the case with interstitial pneumonia (G3), everolimus treatment was interrupted and prednisone was started at a dose of 1,000 mg daily by intravenous injection for 3 days and was then gradually tapered over several weeks. The dose was reduced by $5 \mathrm{mg}$ every 2 weeks and then by $5 \mathrm{mg}$ every week until discontinuation. According to the guideline, we can readminister everolimus at $5 \mathrm{mg}$ only when the benefits obtained outweigh the adverse events. However, the disease state of interstitial pneumonia worsened with treatment and necessitated the introduction of chemotherapy in some patients.

\section{Discussion}

\section{Indications for Everolimus Based on Sensitivity to Endocrine Therapy}

Endocrine therapy sensitivity may originally have been favorable in patients in whom the disease recurred after completion of postoperative endocrine therapy and those receiving prolonged endocrine therapy [4]. It might be important to assess the presence or absence of endocrine therapy sensitivity based on the history of treatments given prior to everolimus administration in each patient, and to then select patients based on whether they had become resistant to endocrine therapy.

For example, in 2 patients at our hospital, the therapeutic effect on recurrence within 2 years after surgery (Table 1b) could not be determined due to adverse effects having developed immediately after administration which necessitated discontinuation. Moreover, the disease state worsened during the drug withdrawal period and treatment could not be restarted in either patient. Endocrine therapy sensitivity may originally have been weak in patients with early recurrence and recurrence during adjuvant therapy, and the speed of disease progression and adverse events are difficult to control in such cases. Based on the experience at our hospital, chemotherapy should have been the first choice for these patients.

\section{Indications for Everolimus Based on Prior Treatment History}

In a clinical study focusing on the number of previous treatments, the median PFS tended to be longer in patients who received everolimus in combination therapy regimens as the first and second lines than in those who received it in later treatment lines [5]. 
In our clinical experience, everolimus was frequently introduced in patients with favorable general conditions at the time that the indication was approved, even when the administration was a late treatment line. All patients previously treated with exemestane acquired SD or a better therapeutic effect. The BRAWO study [5] detected no significant difference in PFS between patients with and without previous exemestane treatment.

The study at our hospital suggested that everolimus is a therapeutic option even for patients who have received several prior treatments and those with a history of prior exemestane treatment.

\section{Indications for Everolimus Depended on Metastatic Sites}

On subgroup analysis in the BOLERO-2 study, the PFS-extending effect was superior in the everolimus treatment group regardless of the presence or absence of metastatic lesions, such as visceral organ and bone metastases [6]. In a study on bone metastasis, the time until transition to PD was extended in the group concomitantly treated with everolimus [6], suggesting that mTOR protein is involved in the survival of osteoclasts [7] and that its inhibition suppresses bone absorption, thereby possibly suppressing the advancement of bone metastatic lesions $[8,9]$. In our clinical experience, the difference in therapeutic effects between patients with and without bone metastasis was not clear. However, considering the biology of breast cancer and bone metastasis, for the aforementioned reasons, it is possible that everolimus should be the treatment of choice for those with bone metastasis. We will accumulate further clinical data and reports in efforts to address these issues.

\section{Indications for Everolimus in Consideration of Quality of Life}

At our hospital, S1 was administered to 4 patients as a pretreatment before everolimus administration. The S1 was administered taking into consideration adverse events associated with everolimus which occur in some patients.

Since noninferiority of S1 in overall survival time as compared with taxane-based drugs has been reported for patients judged to have endocrine therapy-resistant disease without life-threatening metastasis [10], S1 is readily included among the treatment options for maintenance of quality of life (QOL). Similarly, an oral FU preparation, capecitabine, is more likely to be a better option than other chemotherapies with regard to preserving QOL. A clinical study, BOLER0-6 [11], comparing capecitabine with everolimus + exemestane is underway and the results obtained to date are very interesting, possibly serving as a reference for treatment selection in the future.

Considering the importance of QOL maintenance, many clinicians avoid using everolimus because of the associated adverse events. Thus, we considered the following countermeasures against adverse events.

\section{Preventive Treatment for Expected Adverse Events before Stating Everolimus}

In patients who developed stomatitis, reinitiation and continuation of treatment were possible with dose reduction or temporary withdrawal of the drug. A randomized study (Oral Care-BC) designed to investigate whether the stomatitis incidence can be reduced with oral cavity management by dental oral surgeons is now underway [12]. We have not worked with dental oral surgeons at our hospital, but it may be necessary to investigate periodic intervention depending on the results of this study. 
In the BOLERO-2 study, the interstitial pneumonia (noninfectious lung-related adverse event) incidence was $12 \%$, and grade 3 and 4 cases accounted for 3 and $0 \%$, respectively [3].

Two developmental mechanisms have been proposed for drug-induced interstitial pneumonia. One mechanism involves an autoimmunity-associated delayed-type hypersensitivity reaction and the other interference by an immunosuppressor with the recovery/repair mechanism when certain types of injury develop in the lung, such as lung metastasis and pneumonia. The risk factors have not yet been clearly identified, but meticulous observation is necessary for high-risk patients with pulmonary lesions before treatment, such as interstitial shadows, previous radiotherapy, and multiple lung metastases [13].

Regarding the mechanisms underlying the high incidence of grade 3 interstitial pneumonia, it is possible that subjective symptoms were underestimated. Activities of daily living were relatively low in 2 patients who had compression fractures due to bone metastasis. Subjective symptoms were underestimated because the patients did not readily sense dyspnea on exertion, and the pneumonia may have been discovered at an advanced stage.

Based on this experience, we decided to more closely evaluate chest CT images before treatment. We will also periodically examine the lungs using chest radiography and compare the findings with those prior to administration (Table $2 \mathrm{~b}$ ). Periodic evaluation by chest CT may also be necessary for high-risk patients with pulmonary lesions, such as the group described above. Although KL6, serving as tumor marker, is already elevated before initiation of everolimus treatment in many patients, we plan to confirm the level before administration as the baseline to monitor variations along with symptoms. In addition, for difficult-to-diagnose cases, we breast oncologists consult with pulmonologists in the early phase and treat these patients based on the examinations conducted at both departments.

\section{Conclusion}

The indications for and problems with everolimus were investigated based on the effects and adverse events in patients treated with everolimus at our hospital. SD or better outcomes were achieved in more than $50 \%$ of the patients, showing that the therapeutic effect of this drug is relatively favorable. To most effectively use everolimus, it may be important to not only select patients for whom this treatment would be beneficial but to aggressively manage adverse events, allowing administration to be continued.

\section{Statement of Ethics}

The authors have no ethical conflicts to disclose.

\section{Disclosure Statement}

The authors have no conflicts of interest to declare. 


\section{References}

1 Ellis M. Overcoming endocrine therapy resistance by signal transduction inhibition. Oncologist. 2004;9 Suppl 3:20-6.

2 Shin-ichi H, Kimura M. Resistant mechanisms of aromatase inhibitors in breast cancer. J-STAGE. 2015;32(2):68-73.

3 Baselga J, Campone M, Piccart M, Burris HA 3rd, Rugo HS, Sahmoud T, et al. Everolimus in postmenopausal hormone-receptor-positive advanced breast cancer. N Engl J Med. 2012 Feb;366(6):520-9.

4 Piccart M. Advance in ET and ET-resistance. 2nd International Consensus Conference for Advanced Breast Cancer (ABC2); 2013 Nov.

5 Jackisch C, Grischke EM, Schneeweiss A, Decker T, Uleer C, Förster F, et al. Subgroup analysis of efficacy in the routine treatment - Results of the 2 nd interim analysis of BRAWO, the non-interventional trial "Breast Cancer Treatment with Everolimus and Exemestane for HR+ Women". Cancer Res. 2015 May;75(9 suppl):P5-19-12.

6 Yardley DA, Noguchi S, Pritchard KI, Burris HA 3rd, Baselga J, Gnant M, et al. Everolimus plus exemestane in postmenopausal patients with HR(+) breast cancer: BOLERO-2 final progression-free survival analysis. Adv Ther. 2013 Oct;30(10):870-84.

7 Gnant M, Baselga J, Rugo HS, Noguchi S, Burris HA, Piccart M, et al. Effect of everolimus on bone marker levels and progressive disease in bone in BOLERO-2. J Natl Cancer Inst. 2013 May;105(9):654-63.

8 Ory B, Moriceau G, Redini F, Heymann D. mTOR inhibitors (rapamycin and its derivatives) and nitrogen containing bisphosphonates: bi-functional compounds for the treatment of bone tumours. Curr Med Chem. 2007;14(13):1381-7.

9 Glantschnig H, Fisher JE, Wesolowski G, Rodan GA, Reszka AA. M-CSF, TNFalpha and RANK ligand promote osteoclast survival by signaling through mTOR/S6 kinase. Cell Death Differ. 2003 Oct;10(10):1165-77.

10 Hara F, Matsubara N, Saito T, Takano T, Park Y, Toyama T, et al. Randomized phase III trial of taxane versus TS-1 as first line chemotherapy for metastatic breast cancer (SELECT BC: CSPOR-MBC01). SABCS; 2014. P310-01.

11 Bent E, Guy H, Maria J, et al. BOLERO-6 Phase II study of everolimus plus exemestane versus everolimus or capecitabine monotherapy in HR+, HER2- advanced breast cancer. 2013 ASCO Annual Meeting Abstr. 6610.

12 Niikura N, Ota Y, Hayashi N, Naito M, Kashiwabara K, Watanabe K et al. Evaluation of oral care to prevent oral mucositis in estrogen receptor-positive metastatic breast cancer patients treated with everolimus (Oral Care-BC): randomized controlled phase III trial. Jpn J Clin Oncol. 2016 Sep;46(9):879-82.

13 Yoshinobu H. Annual Review KOKYUKI. 2013. pp. 125-30. Japanese. 


\section{Case Reports in Oncology}

\section{Case Rep Oncol 2018;11:511-520}

Yoshida-Ichikawa et al.: Overcoming the Adverse Effects of Everolimus to Achieve Maximum Efficacy in the Treatment of Inoperable Breast Cancer

\section{Previous line}

of all therapy

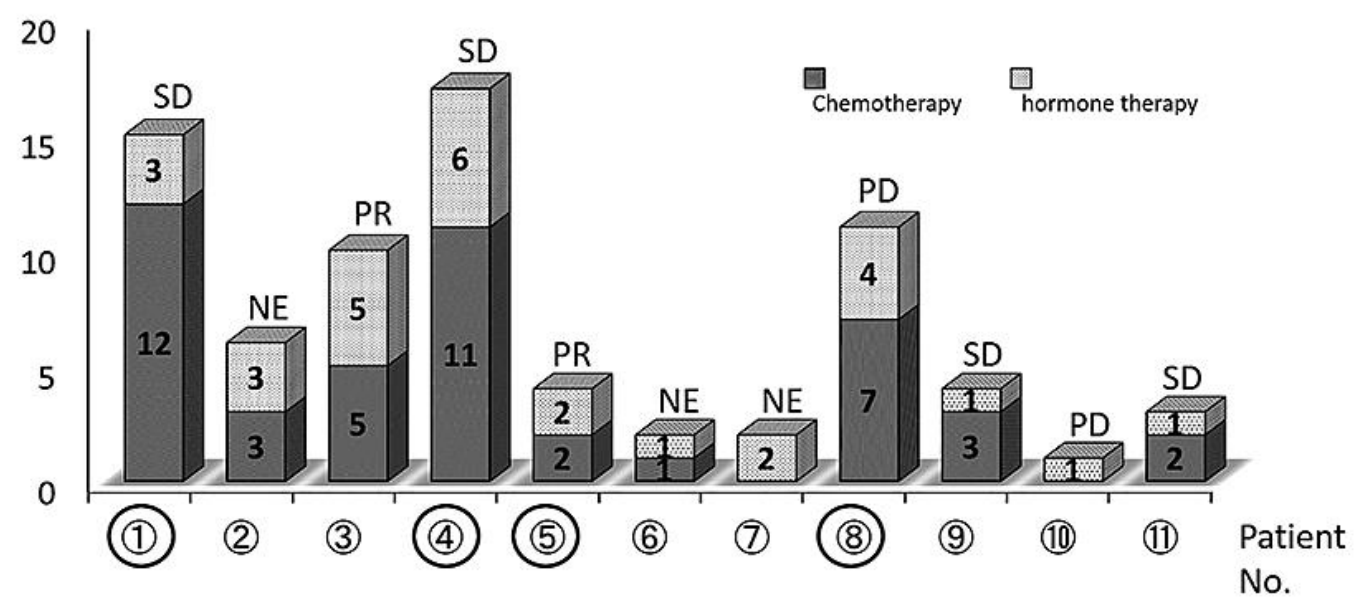

Fig. 1. Details of previous treatments in each patient and therapeutic effects. The patients are listed in order of the time of treatment initiation. Bold circles represent patients in whom exemestane was included in the previous treatment regimen. 
Yoshida-Ichikawa et al.: Overcoming the Adverse Effects of Everolimus to Achieve

Maximum Efficacy in the Treatment of Inoperable Breast Cancer

Table 1. Patient background and maximum therapeutic effect in each patient

a Patient background

$\begin{array}{ll}\text { Median age (range), years } & 58 \\ \text { Stage at diagnosis } & \\ \text { IIA } & 2 \\ \text { IIB } & 4 \\ \text { IIIA } & 1 \\ \text { IIIC } & 1 \\ \text { IV } & 3 \\ \text { Hormone receptor status } & \\ \quad \text { ER+/PgR- } & 3 \\ \text { ER+/PgR+ } & 8 \\ \text { Disease sites at baseline } & \\ \quad \text { Bone only } & 2 \\ \quad \text { Visceral organs only (liver, lung) } & 3 \\ \quad \text { Visceral organs and bone } & 5 \\ \text { Lymph node } & 1\end{array}$

b Maximum therapeutic effect in each patient

PR + long SD SD PD Inevaluable

\begin{tabular}{lllll}
\hline $\begin{array}{l}\text { Disease-free interval, years } \\
\geq 5\end{array}$ & 0 & 2 & 1 & 0 \\
$2-5$ & 1 & 1 & 0 & 1 \\
$<2$ & 0 & 0 & 0 & 2
\end{tabular}

Previous lines of all therapies in the metastatic setting

$>6$
5
4
3
2

2
1
0
0
0

No

2
1
0
0
0

$\begin{array}{lll}1 & 1 & 1\end{array}$

$\begin{array}{lll}1 & 0 & 0\end{array}$

$\begin{array}{lll}1 & 0 & 1\end{array}$

$\begin{array}{lll}0 & 0 & 1\end{array}$

Previous exemestane treatment

Yes
No

Previous lines of hormone therapy

$>5$
4
3
2
1

$>5$
4

3

2

1

Previous chemotherapy

Yes

No

Disease sites at baseline

Bone only

Bone and visceral organs

Visceral organs only

Lymph node

$\begin{array}{lll}0 & 1 & 0\end{array}$

2

$\begin{array}{lll}1 & 1 & 0\end{array}$

$12 \quad 2 \quad 1 \quad 3$

$\begin{array}{llll}1 & 1 & 0 & 0 \\ 0 & 0 & 1 & 0 \\ 1 & 0 & 0 & 1 \\ 1 & 0 & 0 & 1 \\ 0 & 2 & 1 & 1 \\ 3 & & & \\ 0 & 3 & 1 & 2 \\ & 0 & 1 & 1 \\ 0 & & & \\ 1 & 0 & 1 & 1 \\ 1 & 2 & 1 & 1 \\ 1 & 1 & 0 & 1 \\ & 0 & 0 & 0\end{array}$


Table 2. Adverse events and periodic test items

a Adverse events

\begin{tabular}{lccc}
\hline & Grade 1 & Grade 2 & Grade 3 \\
\hline Thrombocytopenia $(n=2)$ & $1(9 \%)$ & $1(9 \%)$ & \\
Interstitial pneumonia $(n=4)$ & $1(9 \%)$ & & $3(27 \%)$ \\
Stomatitis $(n=3)$ & $1(9 \%)$ & $2(18 \%)$ & \\
Diabetes mellitus $(n=2)$ & $1(18 \%)$ & & \\
Skin disorder $(n=1)$ & $18 \%)$ & \\
\hline
\end{tabular}

b Periodic test items

\begin{tabular}{lll}
\hline & $\begin{array}{l}\text { Inspection } \\
\text { item in advance }\end{array}$ & $\begin{array}{l}\text { Periodic } \\
\text { inspection item }\end{array}$ \\
\hline $\begin{array}{l}\text { Hematological examination } \\
\text { Serum biochemical examination (e.g., liver/kidney } \quad\end{array}$ & 0 \\
$\quad$ function, Tchol, TG, blood glucose, Hb1c) & 0 & \\
KL6 & 0 & $\triangle$ \\
Chest CT & 0 & \\
Chest X-ray & 0 & 0 \\
Hepatitis B virus & 0 & \\
\hline
\end{tabular}

Chest CT and KL6 measurement were performed in correspondence to symptoms. Tchol, total cholesterol; TG, triglycerides. 